\title{
The relation between cortisol and functional connectivity in people with and without stress-sensitive epilepsy
}

Jonas M. den Heijer ${ }^{1}$, Willem M. Otte 2,3,4, Eric van Diessen ${ }^{2}$, Jolien S. van Campen ${ }^{2,5}$, E. Lorraine Hompe ${ }^{1}$, Floor E. Jansen ${ }^{2}$, Marian Joels, ${ }^{5,6}$, Kees P.J. Braun ${ }^{2}$, Josemir W. Sander ${ }^{4,7}$ and Maeike Zijlmans ${ }^{2,4}$

${ }^{1}$ University Medical Center Utrecht, Utrecht, The Netherlands

${ }^{2}$ Department of Pediatric Neurology, Brain Center Rudolf Magnus, University Medical Center Utrecht, Utrecht, The Netherlands

${ }^{3}$ Biomedical MR Imaging and Spectroscopy group, Center for Image Sciences, University Medical Center Utrecht, Utrecht, The Netherlands

${ }^{4}$ Stichting Epilepsie Instellingen Nederland (SEIN), Heemstede, The Netherlands

${ }^{5}$ Department of Translational Neuroscience, Brain Center Rudolf Magnus, University Medical Center Utrecht, The Netherlands

${ }^{6}$ University Medical Center Groningen, Groningen, The Netherlands

${ }^{7} \mathrm{NIHR}$ University College London Hospitals Biomedical Research Centre, UCL Institute of Neurology, Queen Square, London WC1N 3BG \& Epilepsy Society, Chalfont St Peter, SL9 ORJ, United Kingdom

Running title: Epilepsy functional network and stress

Keywords: corticosteroid; glucocorticoid; adrenal cortex; connectome; epileptiform discharges; spikes; spike-wave complex;

Number of text pages: 12

Number of words (summary): 293

Number of words (main text): 3939

Number of tables (main and supplementary): main 2, supplementary 0

Number of figures (main and supplementary, and how many are in color): main 2 (color), supplementary 11 (color)

\section{Key Point Box}

- Relationship between cortisol and functional brain connectivity differs between people with and those without stress-sensitive seizures

- Cortisol might affect interictal discharges through an effect on global functional connectivity

- People with stress-sensitive seizures might have a different brain response to stress-hormones 


\section{Summary}

Objective: The most common reported seizure-precipitant is stress. We recently showed a biological basis for stress-sensitivity of seizures: cortisol levels in people with stress-sensitive epilepsy correlated with focal interictal epileptic discharges (IEDs) on EEG. In the current study we aimed to determine whether the effect of cortisol on the epileptic brain is global or focal, and whether cortisol affects all brains or just those of stress-sensitive people. As epilepsy is associated with changes in functional brain connectivity, we studied the relationship between cortisol and changes in global and focal (node-centered) functional connectivity measures for individuals with stress-sensitive and non-stress-sensitive epilepsy.

Methods: Seventeen people with epilepsy underwent long-term (>24h) EEG recording. During the first 5 hours after waking saliva was collected every 15 minutes for cortisol measurements. Theta-band functional connectivity was assessed for every 15 minutes of the recording. We calculated the average phase-lag index (PLI) between channel pairs as a measure of global functional connectivity. We used network Strength, the averaged PLI per channel, as focal functional connectivity measure. We correlated cortisol, global and focal functional connectivity (strength) with IED frequency using linear mixed models. Analyses were split for people with and without seizure-susceptibility to stress.

Results: Cortisol was negatively correlated with global functional connectivity in people with stress-sensitive seizures (estimate -0.0020; $p<0.01$ ), while not in those without stress-sensitivity (estimate $-0.0003 ; p=0.46$ ). This relationship was irrespective of the presence of IEDs on a channel (channels without IEDs and stresssensitivity: estimate $-0.0019 ; p<0.01$, non-stress-sensitive -0.0003; $p=0.41$ ). Global and focal functional connectivity were negatively correlated with IED frequency, irrespective of stress-sensitivity of seizures or channel type.

Significance: People with stress-sensitive epilepsy have a different whole brain neuronal response to cortisol than people with non-stress sensitive epilepsy. This offers a basis for understanding seizure genesis in stresssensitive epilepsy, which might require a different treatment approach. 


\section{Introduction}

Many studies have reported stress to be a major seizure-precipitating factor in people with epilepsy. ${ }^{1 ; 2}$ Cortisol is one of the main hormones released by activation of the hypothalamic-pituitary-adrenal (HPA) axis in response to a stressor ${ }^{3}$. People with epilepsy have higher basal levels of cortisol than those without; the level further increases after seizures ${ }^{4 ; 5}$. Increased seizure frequency in people with epilepsy is associated with high cortisol levels ${ }^{5}$. Cortisol is released in ultradian pulses with a 1-2 hour interval 7;8 and the peaks of these cortisol pulses are highest around awakening, decreasing in subsequent hours, which overall results in a circadian release pattern ${ }^{7 ; 9}$. Animal studies have shown a higher susceptibility to seizure induction at the time of day with the highest cortisol secretion ${ }^{10}$. In humans, a well-documented diurnal occurrence of epileptic seizures shows similarities to the circadian rhythm of cortisol ${ }^{11}$. We recently correlated spontaneous ultradian and circadian variations in cortisol concentration with the frequency of interictal epileptiform discharges (IEDs) in individuals with focal epilepsy and found a positive correlation only in people with reported seizure susceptibility to periods of stress ${ }^{12}$. This suggests a neurobiological basis for stress-sensitive epilepsy. An outstanding question is how this interaction between cortisol and the epileptic brain takes place.

The brain is organized and functions as a complex network. This justifies the characterization of the brain with network tools. These tools require two sets of input data to shape the networks: information at the level of nodes and information on the connections between pairs of nodes. In this study we represented the nodes as EEG channels and the connections as the electro cortical synchronization between epochs selected from these channels. These representations provide us with functional networks covering the whole brain ${ }^{13 ;}$

${ }^{14}$. Functional networks are typically characterized with summary metrics quantifying the networks' topology or organization. The topology may range from regular to random and differs across frequency bands. The integration of nodes in the overall network is also an important metric. The functional network during ictal periods in people with epilepsy, compared to interictal states, has consistently been shown to be more regularly organized ${ }^{15-17}$. However, the organization of the interictal network is less clear. A recent metaanalysis suggested a less integrated interictal whole-brain network, particularly in the theta frequency band ${ }^{18}$, but an increase in functional connectivity in different frequency bands was seen in people with epilepsy when compared to healthy controls ${ }^{19 ; 20}$. Functional connectivity allows the assessment of changes globally and focally in the brain.

We hypothesized that cortisol has an effect on the epileptogenic brain, expressed as a change in functional connectivity, which facilitates the generation of IEDs. We wondered whether this effect was indeed present and if so, whether the effect on connectivity was found in all or was restricted to those with stress-sensitive epilepsy and also whether the effect was global (i.e. involving all electrodes) or focal, potentially restricted to 
the brain area producing interictal epileptiform discharges. This comparison can clarify whether cortisol has its effect on all neurons or specifically on the epileptic tissue.

\section{Materials and methods \\ The cohort}

We used a previously reported cohort ${ }^{12}$. It consisted of 23 adults with an established diagnosis of focal epilepsy of variable aetiology (Table 1) who underwent long-term (>24h) EEG recording for either diagnostic purposes or pre-surgical evaluation. Those with frequent seizures (more than once every five hours) or on oral contraceptive (due to its effect on cortisol levels) ${ }^{12}$ were excluded. For the current study, we retrospectively excluded those with a seizure during the five hour of saliva sampling, as seizures result in network alterations, and those with major artifacts in the EEG recording, resulting in 17 people for further analysis. Our ethical committee approved the study and all provided written informed consent.

\section{Data acquisition and selection}

\section{Saliva cortisol}

From awaking, subjects were asked to provide a saliva sample every 15 minutes for the next five hours, resulting in a total of 20 saliva samples per subject. Cortisol concentrations in saliva lag plasma levels by only two to three minutes ${ }^{21}$. A rodent study has shown a strong correlation between fluctuations in peripheral and brain corticosteroid levels 22 .

Sterile Salivettes ${ }^{\circledR}$ (Starstedt, Etten-Leur, the Netherlands) cotton swabs were used and at sampling times the subject chewed one for two minutes. No food or drinks were consumed in the five minutes prior to or during sampling. To avoid contamination of saliva, subjects were asked to rinse their mouth with water five minutes before sampling after any food intake ${ }^{23}$.

Salivettes ${ }^{\circledR}$ were stored at $-9{ }^{\circ} \mathrm{C}$ for one to four days, after which they were centrifuged for 10 minutes at $3000 \mathrm{rpm}$. Storage till further analysis was at $-20{ }^{\circ} \mathrm{C}$. Cortisol levels were measured without extraction with an in-house competitive radio-immunoassay using a polyclonal anticortisol-antibody $(\mathrm{K} 7348)$ and $\left[1,2-{ }^{3} \mathrm{H}(\mathrm{N})\right]-$ hydrocortisone (PerkinElmer NET396250UC) tracer ${ }^{24}$. All samples from a subject were analyzed in the same batch.

\section{Stress-sensitivity of seizures}

Subjects completed a questionnaire, including a 'yes' or 'no' question on whether they experienced more seizures in periods of stress ${ }^{6}$. Subjects who answered with 'yes' were considered to have stress-sensitive seizures. A previous comparative study on questionnaire and diary data regarding stress sensitivity of 
seizures revealed that both methods yield comparable results ${ }^{6}$. Hence, we selected the approach with the lowest burden for participants.

\section{Antiepileptic drug (AED) withdrawal and experienced stress}

In individuals in whom medication was tapered before or during a presurgical 5-day EEG-recording, the experiment was performed on the third and fourth day to minimize interference of change in AED dosage and to minimize the effect of stress caused by the admission. During the time of the experiment, activities were documented every 15 minutes and individuals provided a subjective stress score on a visual analogue scale (VAS) ranging from "no stress" (0) to "the most severe stress I have ever experienced" (10).

\section{EEG and epoch selection}

The EEG was recorded with 32 scalp electrodes, placed according to the 10-20 international system, referred to a G2 electrode (placed between $\mathrm{Cz}$ and Fz) and referenced to an average montage for further analysis. Signals were sampled at $256 \mathrm{~Hz}$, with the exception of four subjects, who were sampled at $1024 \mathrm{~Hz}$. Data of the $1024 \mathrm{~Hz}$ EEGs were downsampled to $256 \mathrm{~Hz}$ using Matlab for further analysis. Only standard electrodes were used for analyses, Fz, F3, F4, F7, F8, Cz, C3, C4, Pz, P3, P4, T3, T4, T5, T6, O1, O2 (Fp1 and Fp2 were excluded due to their high sensitivity to eye-movement artifacts).

We selected 4-second epochs with eyes open, as subjects did not receive specific instructions to close eyes for these diagnostic or pre-surgical recordings. We chose to select epochs of four seconds in length (each containing 1024 samples), to allow for sufficient signal to accurately determine the phase synchronization between channels. The epochs were selected in a time range of 7.5 minutes before to 7.5 minutes after every saliva sampling. Epochs with as few artifacts as possible were selected, according to the following procedure. First we made a preselection of all possibly usable epochs per previously mentioned time range of saliva sampling, excluding evident movement, eye-movement and eye-blink artifacts, wide-spread muscle and epileptiform spikes. Selections with minor artifacts, which we considered usable though suboptimal, were given a special mark (see below). Epochs were visually selected by one of the authors (JdH) and verified by an experienced neurologist (MZ). For the eventual analysis, we chose the four epochs closest to sampling time that were marked as artifact-free. If fewer than four artifact-free epochs were available, we chose the first available epochs from time of sampling that were marked as suboptimal up to a total of four epochs. If within the total time range less than four epochs were available, with a minimum of one epoch, we still considered this a viable timeslot and continued to use these in further analyses. Two randomly-selected epochs of nine subjects are given as examples in the supporting information, published online only.

Selected epochs were exported from Micromed System Evolution Plus. Analysis of functional connectivity was done using Brainwave (ver. 0.9.133.1; authored by CJ Stam) and further statistical analysis using SPSS (ver. 23). 


\section{Interictal epileptiform discharges}

We determined the frequency of interictal epileptiform discharges as previously described ${ }^{12}$. In short, IEDs were defined as spikes or sharp waves (including single spikes, polyspikes, spike-and-slow-wave complexes, sharp-and-slow-wave complexes and single sharp waves) with an evident epileptiform pattern. IEDs were counted per 7.5 minutes and then divided by 7.5 to get an average IED frequency per minute. IED frequency was calculated across channels.

We distinguished channels with and without IEDs per subject, judged by an epileptologist (MZ).

\section{Functional connectivity}

Functional connectivity is based on statistical interdependencies between signals, as reflected by the synchronization of two temporal time-series. The connectivity measure Phase Lag Index (PLI) ${ }^{25}$ was used to compute a functional network from every epoch, based on the synchronization of any pair of electrodes. This index quantifies the phase synchronization of two time-series as the asymmetry of the phase difference distribution of all phase differences $\Delta \Phi\left(t_{k}\right), k=1 \ldots 1024$ in the following way:

$$
\mathbf{P L I}=\left|<\operatorname{sign}\left[\Delta \Phi\left(\mathrm{t}_{\mathrm{k}}\right)\right]>\right|
$$

The PLI ignores zero-phase interactions (at the expense of ignoring true zero phase interactions) to exclude volume conduction as a confounding factor. This index ranges from 0 to 1 , in which a higher number indicates a stronger synchronization, enabling us to create a weighted network. For details see ${ }^{25}$.

We chose to investigate primarily the theta band, but looked at the other frequency bands in a post-hoc analysis, with filters set to: delta $(0.5-4 \mathrm{~Hz})$, theta $(4-8 \mathrm{~Hz})$, alpha ${ }_{1}(8-10.5 \mathrm{~Hz})$, alpha $2(10.5-13 \mathrm{~Hz})$ and beta $(13-20 \mathrm{~Hz})$. Since the EEG signal contained superimposed muscle activity, we chose not to investigate frequencies higher than $20 \mathrm{~Hz}^{26}$.

\section{Network analysis}

We used the global PLI, calculated by averaging all PLIs per channel, to determine global functional connectivity of selected epochs, where a higher PLI indicates a higher global functional connectivity. We used Strength as a node-centered measure of functional connectivity, by averaging the PLI of that certain electrode in relation to all other electrodes. Global PLI and Strength per electrode of the four epochs per time of sampling were averaged.

\section{Statistical analysis}

Time lag analysis

We first explored whether there was a time lag effect between cortisol and PLI by doing a cross correlation with shifts of 15 minutes (for every cortisol sample) as we expected a potential delayed relationship 
between hormonal release and functional brain modifications. We only evaluated positive time shifts, in which cortisol precedes changes in functional connectivity Strength, given the expected directionality of correlations. We did this by averaging the absolute cross correlation coefficient over all subjects per time lag. We would expect a time lag to have a similar biological mechanism over all subjects, therefore we explored whether a significant shift was found over all subjects and continued further analyses applying that time lag. We did not perform a time lag analysis for the possible relationship between PLI and IED frequency, as we did not have a physiological rationale to support such a delay and we aimed to limit the number of comparisons performed.

\section{Cortisol and functional connectivity}

We investigated the relationship between changes in cortisol and average PLI, as proxy for global functional connectivity, to see whether cortisol has an effect on the whole-brain functional network. Due to the interindividual variation in cortisol concentration, we applied a linear mixed model (LMM) with 'subject' as a random factor, including the intercept of the random effects), to correct for this inter-individual variation, and 'cortisol' as fixed factor. Covariance type was set to 'Scaled Identity'. We compared subjects with and without seizure susceptibility to periods of stress, by stratifying our data for both groups. We verified our results with bootstrapping, a statistical test which is less influenced by outliers, to check the robustness of a model. We did this for all following tests.

We investigated the relationship between cortisol and Strength per channel, calculated as the average PLI of that channel with every other channel, with a similar LMM.

To see whether the effect of cortisol on functional connectivity was specific for channels with IEDs, we calculated an average Strength of all channels that contained IEDs for all subjects individually (Supplementary Figure 3). We then grouped these newly calculated PLIs to continue analysis with an LMM. We did the same for all channels without IEDs per subject.

Functional connectivity and interictal epileptiform discharge (IED) frequency

We investigated the relationship between average PLI of the network and IED frequency using a LMM. In accordance with our hypothesis, that a change in functional connectivity facilitates the generation of IEDs, we used IED frequency as the dependent variable, subjects as the random identity factor with intercept included, and PLI as fixed factor. Covariance type was set to 'Scaled Identity'.

To see whether node-centered changes in connectivity might influence the IED frequency, we also looked at Strength per channel in relationship to IED frequency, with similar LMMs.

We analyzed the PLI in relation to IED frequency of subject-specific channels containing IEDs or not (LMM). We considered $\mathrm{p}$-values of $<0.05$ significant. We did not correct for multiple comparisons. 


\section{Results}

\section{Clinical characteristics}

Six individuals were excluded, four due to a seizure during the sampling and two due to excessive electrographic artifacts. Seventeen people (age 39 SD? SE? \pm 15 years, 10 males, 7 females) were included, of whom six reported stress-sensitive seizures (Table 1). In the 17 included subjects, 338 of 340 cortisol samples were successful.

We selected 1070 epochs (average 63 per person; range 38-78). Each individual had on average 18 viable timeslots (minimum 12, maximum 20, total 307), containing at least one epoch. For every viable timeslot we had a cortisol sample, apart from one (subject 8, timeslot 13 ).

The averaged values over all 17 subjects were: cortisol $13.7 \mathrm{nmol} / \mathrm{L}$ (range 4.7 - $35.0 \mathrm{nmol} / \mathrm{L}$, standard deviation (SD) $5.9 \mathrm{nmol} / \mathrm{L}$ ), theta power 0.157 (range $0.057-0.356$, SD 0.060), PLI 0.192 (range 0.135 0.444 , SD 0.038), IED frequency 2.0 per minute $(0.0-40.3$, SD 5.7 per minute).

\section{Time lag analysis}

The highest absolute cross correlation averaged over all subjects (mean $=0.24$ ) was found when cortisol led PLI by two timeslots, meaning PLI based on epochs selected between 22.5 and 37.5 minutes after saliva sampling. We continued analysis with this time lag.

\section{Cortisol and global functional connectivity}

We found a negative association between cortisol and averaged PLI, meaning a decrease in global functional connectivity in the window of $\mathbf{2 2 . 5}$ to $\mathbf{3 7 . 5}$ minutes after cortisol increased. After stratification for stresssensitivity of seizures, this association remained in the group that reported stress-sensitivity (Table 2; Figure 1). After bootstrapping the linear mixed model, as a verification of robustness of the model, significance remained unchanged. Some PLI averages seemed remarkably high; after removal of these epochs from analyses, significance remained unchanged.

\section{Cortisol and Strength per channel}

In all seventeen channels, we found a negative relationship between cortisol and Strength, of which six were significant in the total group (Table 3). After splitting individuals based on their stress sensitivity, the stresssensitive group yielded fourteen channels with a significant relationship while none of the channels in the stress-insensitive group showed a significant relationship (Figure 2).

\section{Cortisol and average Strength of subject-specific channels with and without IEDs}

A significant negative relationship between cortisol and averaged Strength was observed for all subjectspecific channels with IEDs and for all channels without IEDs (Table 2). This suggests that the relationship 
between cortisol and functional connectivity is not specific for brain regions showing IEDS, but the effect on the brain is global. After stratification for seizure susceptibility to stress, only the susceptible group showed a significant relationship. After bootstrapping significance did not change.

\section{Functional connectivity and interictal epileptiform discharge (IED) frequency}

We found a negative relationship between average PLI and IED frequency (time lag 0). After stratification for seizure susceptibility to periods of stress, both groups remained significant (Table 2). After bootstrapping, only the total group remained significant.

In all seventeen channels, we found a negative relationship between Strength (PLI per channel) and the general IED frequency per subject in the same time slot, of which eight channels were significant in the total group. After splitting individuals based on their stress sensitivity, the stress-sensitive group yielded four channels with a significant relationship while four other channels showed a significant relationship in the stress-insensitive group (supplementary Figure 4). The negative relationship between Strength and IED frequency remained for (subject-specific) channels with and without IEDs (table 2). After stratification for seizure susceptibility to stress, only in the group without IEDs, the susceptible subjects marginally lost significance $(p=0.06)$. After bootstrapping, only the total group with Strength of channels with IEDs remained significant.

\section{Discussion}

\section{Cortisol and functional connectivity}

We found a decrease in theta band average PLI with increased cortisol levels in people with stress-sensitive focal epilepsy. A decrease in average PLI reflects a decrease in global functional connectivity. Similarly, when looking at node-centered functional connectivity, we found several channels decreased in strength as cortisol increased. When differentiating between channels with and without IEDs on a subject specific level, the negative correlation between cortisol and Strength persisted in both groups of channels. Functional connectivity negatively correlated with IED frequency, without the differentiation between stress-sensitivity or not. In other words, cortisol in its natural daily rhythm affects global and local functional connectivity measures of the whole brain, irrespective of the irritative zone, in people with stress-sensitive epilepsy, which could facilitate the generation of IEDs and maybe seizures.

Most studies comparing people with epilepsy in the interictal state with healthy controls reported a higher functional connectivity in those with epilepsy ${ }^{19 ; 20 ; 27 ; 28}$. An increase in theta band functional connectivity was shown to be associated with seizure frequency in tumor-related epilepsy ${ }^{29}$. One could speculate that the increased functional connectivity in people with epilepsy is somehow a protective mechanism against seizure generation, which fails in a stress susceptible subset of subjects as cortisol increases. There is 
evidence for the hypothesis that desynchronization takes place accompanied by a reduction of IEDs prior to seizure onset. ${ }^{30}$ In the stress susceptible subset the decrease in global functional connectivity as cortisol increases could be a first expression of susceptibility to seizure generation. It should be noted that any causality, let alone a directionality is still open for debate. It could also be speculated that it is the fluctuation in cortisol that facilitates an effect, irrespective of whether it is an increase or decrease.

In time lag analysis between cortisol and PLI we found an optimal correlation when cortisol leads PLI by 22.5 to 37.5 minutes. A delayed effect of cortisol on PLI might be explained by the delayed cellular effect of cortisol in the brain. Cortisol has rapid and slow effects on neuronal cells, varying from minutes to hours after increase ${ }^{31}$. This time lag, however, is incongruous with the time lag found by Van Campen et al. (2016) where a maximal effect of cortisol on IED frequency was shown in the 15 minutes following saliva sampling. A possible explanation could be an overestimation of the delay on functional connectivity because of the small sample size. In the cross-correlation analyses of cortisol and average PLI, a time lag of 0 and 1 both were significant as well, though the time lag of 2 was slightly stronger. Repetition in a larger cohort would be necessary to confirm this.

Cortisol, functional connectivity, IED frequency and stress-sensitivity of seizures

Some EEG - $\mathrm{fMRI}$ studies have looked at the relationship between IEDs and functional network changes and each of these showed network changes prior to IEDs, suggesting that it might be the change in functional connectivity that facilitates IED ${ }^{32-35}$. Furthermore, a relationship between changes in EEG network topology and the frequency of IEDs was found to exist ${ }^{35}$. Whether IEDs in turn also affect functional connectivity is under debate.

We found a stress-sensitivity driven correlation between cortisol and IED frequency and functional connectivity and an overall correlation between functional connectivity and IED frequency, irrespective of stress-sensitivity. Thus, we hypothesize that the influence of cortisol on global functional connectivity mediates the effect of cortisol on IED frequency in people with seizures susceptible to stress. We acknowledge that our results cannot be conclusive in the debate of the directionality of the relationship between functional connectivity and IED generation.

\section{Study design and limitations}

Our study has some limitations. Firstly, we cannot generalize to resting state EEG recordings ${ }^{13}$. Subjects did not receive instructions to (periodically) keep their eyes closed, thus we had to select eyes open epochs. The eyes closed condition is more stable over sessions when quantifying EEG parameters than the eyes open condition ${ }^{36}$. As subjects were free to move, the EEGs also contained many movement, muscle and blinking artifacts. We tried to overcome this by a careful epoch selection procedure. 
Secondly, epochs were restricted to a length of 4 seconds to keep this identical over all subjects, as epoch length can influence the connectivity measure ${ }^{37}$. It was recently demonstrated that PLI might be overestimated in epochs shorter than twelve seconds, though the variance seemed similar at four seconds and should thus be reliable to register a change in PLI in successive measurements ${ }^{38}$. The equipment used recorded the EEGs at $256 \mathrm{~Hz}$, also limiting the sample frequency, though it is not completely clear how exactly the sample frequency influences connectivity measures ${ }^{39}$.

Retrospectively, we reassessed the selected epochs by random sampling. Artifacts in the used epochs were mostly caused by muscle activity, which largely influences frequencies above $20 \mathrm{~Hz}{ }^{26}$ and are thus unlikely to influence theta band results. There were no eye-blinks and only sporadic eye-movement artifacts, without an evident difference in incidence between beginning and ending of registration. Any influence of these would be approximately similar over the whole registration and should thus have no effect on our long-term analysis. Subjects could be more active in the course of the day influencing successive measurements, though this would not explain the differences we found between subjects reporting stress susceptibility or not.

Thirdly, saliva sampling is widely used for cortisol measurements, though it is limited in registering shortterm changes. We expected to find a clear pulsatile pattern with measurements with a 15 minutes interval, as the ultradian pattern is known to be $1-2$ hours $7 ; 8 ; 40$. The patterns showed a large inter-individual variation and no obvious ultradian pulsatile pattern in all people, though all showed a descending trend in concentration over the five hours measured immediately after awakening.

Fourthly, the experience of an epilepsy monitoring unit admission could induce stress, although measurements were performed at day 3-4 after admission, so acute admission stress is unlikely. Reported subjective stress scales did not show unexplained fluctuations ${ }^{12}$.

Fifthly, since the underlying substrates were very diverse, the brain lesions could confound the measures of synchrony. However, we found both global and local effects and these local effects were not restricted to channels showing IEDs. Also, we compared all channels with IEDs to all channels without IEDs and found no clear difference.

Lastly, we did not have a control group, and neither has the influence of cortisol on functional connectivity in the healthy brain yet been investigated. Despite these limitations, we consider this investigation to be a valuable incentive for further exploration of graph theoretical changes in the epileptic brain caused by cortisol and stress, and their consequence for seizure susceptibility.

\section{Future work and conclusion}

Considering the cortisol peak around awakening, it would be interesting to investigate further how these findings relate to specific epilepsy syndromes whose seizure occurrence can have strong relationships with the sleep-wake cycle, such as juvenile myoclonic epilepsy and nocturnal frontal lobe epilepsy. 
Future study in larger cohorts, including healthy subjects and comparing cortisol levels to seizures and other EEG phenomena is needed to help interpret these findings. The effect of cortisol on the brain in people with stress-sensitive epilepsy underlines that this entity differs from non-stress-sensitive epilepsy in its systemlevel etiology. This group might profit from a different therapeutical strategy which requires large cohort therapy-response studies.

\section{Acknowledgement and Disclosures}

J.v.C. was supported by the UMC Utrecht Alexandre Suerman Stipendium, M.Z. was supported by the Rudolf Magnus Young Talent Fellowship and ZonMW veni grant no.91615149. W.M.O. was supported by the Netherlands Organization for Scientific Research [veni 016.168.038] and the Dutch Brain Foundation [F2014(1)-06]. JWS is based at the UCLH/UCL Bio-Medical Research Centre which received a proportion of funding from the Department of Health's NIHR Biomedical Research Centres funding scheme; his current position is endowed by UK Epilepsy Society and he has received research support from the Marvin Weil Epilepsy Research Fund. His department has received research grants from GSK, Eisai, UCB, EU, Dutch Epilepsy Funds, WHO and UK Epilepsy Society. He has received honoraria from UCB, Eisai, GSK, Lundbeck and Teva. The remaining authors have no conflict of interest.

\section{Ethical Publication Statement}

We confirm that we have read the Journal's position on issues involved in ethical publication and affirm that this report is consistent with those guidelines. 


\section{References}

1. Maguire J, Salpekar JA. Stress, seizures, and hypothalamic-pituitary-adrenal axis targets for the treatment of epilepsy. Epilepsy and Behvior 2012;26 SRC - 352-362.

2. van Campen JS, Jansen FE, de Graan PNE, et al. Early life stress in epilepsy: a seizure precipitant and risk factor for epileptogenesis. Epilepsy \& behavior : E\&B 2014;38:160-171.

3. Kloet ER, Joels M, Holsboer F. Stress and the brain: from adaptation to disease. Nat Rev Neurosci 2005;6 SRC - G:463-475.

4. Culebras A, Miller M, Bertram L, et al. Differential response of growth hormone, cortisol, and prolactin to seizures and to stress. Epilepsia 1987;28 SRC - 564-570.

5. Galimberti CA, Magri F, Copello F, et al. Seizure frequency and cortisol and dehydroepiandrosterone sulfate (DHEAS) levels in women with epilepsy receiving antiepileptic drug treatment. Epilepsia 2005;46 SRC - 517-523.

6. van Campen JS, Jansen FE, Pet MA, et al. Relation between stress-precipitated seizures and the stress response in childhood epilepsy. Brain : a journal of neurology 2015;138:2234-2248.

7. Lightman SL, Conway-Campbell BL. The crucial role of pulsatile activity of the HPA axis for continuous dynamic equilibration. Nat Rev Neurosci 2010;11:710-718.

8. Veldhuis JD, Iranmanesh A, Johnson ML, et al. Amplitude, but not frequency, modulation of adrenocorticotropin secretory bursts gives rise to the nyctohemeral rhythm of the corticotropic axis in man. Endocrinol Metab 1990;71 SRC - 452-463.

9. Dickmeis T. Glucocorticoids and the circadian clock. The Journal of endocrinology 2009;200 SRC -:3-22.

10. Ehlers CL, Killam EK. Circadian periodicity of brain activity and urinary excretion in the epileptic baboon. Am 1980;239 SRC -:R35-41.

11. van Campen JS, Valentijn FA, Jansen FE, et al. Seizure occurrence and the circadian rhythm of cortisol: a systematic review. Epilepsy \& behavior : E\&B 2015;47:132-137.

12. van Campen JS, Hompe EL, Jansen FE, et al. Cortisol fluctuations relate to interictal epileptiform discharges in stress sensitive epilepsy. Brain : a journal of neurology 2016;139:1673-1679.

13. Bullmore E, Sporns O. Complex brain networks: graph theoretical analysis of structural and functional systems. Nat Rev Neurosci 2009;10 SRC - 186-198.

14. Stam CJ. Characterization of anatomical and functional connectivity in the brain: a complex networks perspective. Int 2010;77 SRC - 186-194.

15. Gupta D, Ossenblok $P$, van Luijtelaar $G$. Spacetime network connectivity and cortical activations preceding spike wave discharges in human absence epilepsy: a MEG study. Med Biol Eng Comput 2011;49:555-565

16. Ponten SC, Bartolomei F, Stam CJ. Small-world networks and epilepsy: graph theoretical analysis of intracerebrally recorded mesial temporal lobe seizures. Clin Neurophysiol 2007;118 SRC -:918-927.

17. Ponten SC, Douw L, Bartolomei F, et al. Indications for network regularization during absence seizures: weighted and unweighted graph theoretical analyses. Exp Neurol 2009;217:197-204.

18. van Diessen E, Zweiphenning WJEM, Jansen FE, et al. Brain Network Organization in Focal Epilepsy: A Systematic Review and Meta-Analysis. PloS one 2014;9:e114606.

19. Niso G, Carrasco S, Gudín M, et al. What graph theory actually tells us about resting state interictal MEG epileptic activity. Neuroimage Clin 2015;8 SRC - G:503-515.

20. Wang B, Meng L. Functional brain network alterations in epilepsy: A magnetoencephalography study. Epilepsy research 2016;126:62-69.

21. Kirschbaum, Hellhammer. Encyclopedia of Stress: A-D 2000:379-383.

22. Qian X, Droste SK, Lightman SL, et al. Circadian and ultradian rhythms of free glucocorticoid hormone are highly synchronized between the blood, the subcutaneous tissue, and the brain. Endocrinology 2012;153:4346-4353.

23. Gröschl M, Knerr I, Topf H-G, et al. Endocrine responses to the oral ingestion of a physiological dose of essential amino acids in humans. The Journal of endocrinology 2003;179:237-244. 
24. van den Bos R, Taris R, Scheppink B, et al. Salivary cortisol and alpha-amylase levels during an assessment procedure correlate differently with risk-taking measures in male and female police recruits. Frontiers in behavioral neuroscience 2013;7:219.

25. Stam CJ, Nolte G, Daffertshofer A. Phase lag index: assessment of functional connectivity from multi channel EEG and MEG with diminished bias fromcommon sources. HumBrainMapp 2007;28 SRC 1178-1193.

26. Whitham EM, Pope KJ, Fitzgibbon SP, et al. Scalp electrical recording during paralysis: quantitative evidence that EEG frequencies above $20 \mathrm{~Hz}$ are contaminated by EMG. Clinical neurophysiology: official journal of the International Federation of Clinical Neurophysiology 2007;118:1877-1888.

27. Douw L, de Groot M, van Dellen E, et al. 'Functional connectivity' is a sensitive predictor of epilepsy diagnosis after the first seizure. PLo e10839 2010;5 SRC - G.

28. Horstmann M-TT, Bialonski S, Noennig N, et al. State dependent properties of epileptic brain networks: comparative graph-theoretical analyses of simultaneously recorded EEG and MEG. Clin Neurophysiol 2010;121 SRC -:172-185.

29. Douw L, van Dellen E, de Groot M, et al. Epilepsy is related to theta band brain connectivity and network topology in brain tumor patients. 103 2010;11 SRC -:103.

30. Cash S. Status epilepticus as a system disturbance: Is status epilepticus due to synchronization or desynchronization? Epilepsia 2013;54:37-39

31. Joels M. Stress, the hippocampus, and epilepsy. Epilepsia 2009;50:586-597.

32. Coito A, Genetti M, Pittau F, et al. Altered directed functional connectivity in temporal lobe epilepsy in the absence of interictal spikes: A high density EEG study. Epilepsia 2016;57:402-411.

33. Fahoum F, Lopes R, Pittau F, et al. Widespread epileptic networks in focal epilepsies: EEG-fMRI study. Epilepsia 2012;53:1618-1627.

34. Fahoum F, Zelmann R, Tyvaert L, et al. Epileptic discharges affect the default mode network--FMRI and intracerebral EEG evidence. PloS one 2013;8:e68038.

35. Ibrahim GM, Cassel D, Morgan BR, et al. Resilience of developing brain networks to interictal epileptiform discharges is associated with cognitive outcome. Brain : a journal of neurology 2014;137:2690-2702.

36. Corsi-Cabrera M, Galindo-Vilchis L, Rio-Portilla Y, et al. del- Withinsubject reliability and inter-session stability of EEG power and coherent activity in women evaluated monthly over nine months. Clin Neurophysiol 2007;118 SRC -:9-21.

37. van Dellen E, van der Kooi AW, Numan T, et al. Decreased functional connectivity and disturbed directionality of information flow in the electroencephalography of intensive care unit patients with delirium after cardiac surgery. Anesthesiology 2014;121:328-335.

38. Fraschini M, Demuru M, Crobe A, et al. The effect of epoch length on estimated EEG functional connectivity and brain network organisation. Journal of neural engineering 2016;13:036015.

39. van Diessen E, Numan T, van Dellen E, et al. Opportunities and methodological challenges in EEG and MEG resting state functional brain network research. Clinical neurophysiology : official journal of the International Federation of Clinical Neurophysiology 2015;126:1468-1481.

40. Hellman L, Nakada F, Curti J, et al. Cortisol is secreted episodically by normal man. The Journal of clinical endocrinology and metabolism 1970;30:411-422. 
Tables

\begin{tabular}{|c|c|c|c|c|c|c|c|c|c|c|c|}
\hline subject & sex & $\begin{array}{l}\text { age } \\
(\mathrm{yr})\end{array}$ & $\begin{array}{c}\text { age at } \\
\text { onset } \\
\text { (yr) }\end{array}$ & $\begin{array}{l}\text { seizure } \\
\text { frequency (per } \\
\text { month) }\end{array}$ & hemisphere & localisation & etiology & $\begin{array}{l}\text { stress- } \\
\text { sensitive } \\
\text { seizures }\end{array}$ & $\begin{array}{l}\text { EEG } \\
\text { duration }\end{array}$ & AED use & $\begin{array}{l}\text { AED } \\
\text { tapering }\end{array}$ \\
\hline 3 & $f$ & 48 & 25 & 0.1 & $\bar{L}$ & Frontal & hemorrhagic infarct & + & 5 day & LTG, LEV, CLB & + \\
\hline 6 & $\mathrm{~m}$ & 59 & 3 & 10 & L & Temporal & MTS & + & 5 day & LEV, LTG & + \\
\hline 7 & $\mathrm{~m}$ & 43 & 0 & 2 & $\mathrm{R}$ & Temporal & MTS & - & 5 day & VPA, CBZ & + \\
\hline 8 & $\mathrm{~m}$ & 22 & 18 & 20 & $\mathrm{~L}$ & Temporal & $\begin{array}{l}\text { hippocampal developmental } \\
\text { disorder }\end{array}$ & + & $24 \mathrm{~h}$ & none & - \\
\hline 9 & $\mathrm{f}$ & 21 & 11 & 150 & $R$ & Temporal & MTS & + & 5 day & CLB, LTG, LEV & + \\
\hline 10 & $\mathrm{~m}$ & 64 & 20 & 3 & $\mathrm{R}$ & Temporal & MTS & - & 5 day & LEV, LSM & + \\
\hline 11 & $\mathrm{~m}$ & 23 & 21 & 0.4 & L & Temporal & Unknown & + & $24 \mathrm{~h}$ & LEV & - \\
\hline 12 & $\mathrm{f}$ & 52 & 32 & $3-4$ & $\mathrm{R}$ & Frontal & Unknown & - & 5 day & TPM, OCB & - \\
\hline 14 & $\mathrm{~m}$ & 42 & 8 & 3 & $\mathrm{R}$ & Temporal & MTS & - & 5 day & CBZ, LEV, PGB & + \\
\hline 15 & $\mathrm{f}$ & 59 & 21 & 6 & $\mathrm{R}$ & Temporal & Unknown & - & 5 day & PHT, PB, LTG & + \\
\hline 17 & $\mathrm{~m}$ & 23 & 16 & $3-4$ & $\mathrm{R}$ & Temporal & MTS & + & 5 day & CBZ & + \\
\hline 18 & $\mathrm{~m}$ & 30 & 25 & $1-2$ & $R$ & Temporal & Unknown & - & 5 day & CBZ & + \\
\hline 19 & $\mathrm{f}$ & 34 & 15 & $3-4$ & $\mathrm{R}$ & Temporal & Unknown & - & 5 day & LTG & + \\
\hline 20 & $\mathrm{~m}$ & 23 & 5 & 5 & $R+L$ & Frontal & Unknown & - & $24 \mathrm{~h}$ & none & - \\
\hline 21 & $\mathrm{~m}$ & 46 & 26 & 2 & $\mathrm{R}$ & Frontal & polymicrogyria + heterotopia & - & 5 day & LTG, CBZ & + \\
\hline 22 & $\mathrm{f}$ & 52 & 23 & 2 & $\mathrm{R}$ & Temporal & MTS & - & 5 day & LTG, OCB & + \\
\hline 23 & f & 26 & 2 & 1 & L & Frontal & cortical dysplasia & - & $24 \mathrm{~h}$ & CBZ, PHT & + \\
\hline
\end{tabular}

Table 1. Individual characteristics

yr years; stress-sensitive seizures self-reported increase in seizure frequency in periods of stress; $E E G$ electroencephalogram; $A E D$ antiepileptic drug; $m$ male; $f$ female; $L$ left; $R$ right; MTS mesiotemporal sclerosis; + yes; - no; $h$ hour; CBZ carbamazepine; CLB clobazam; GBP gabapentin; LEV levetiracetam; LTG lamotrigine; $L S M$ lacosamide; OCB

oxcarbazepine; $P B$ phenobarbital; $P G B$ pregabalin; $P H T$ phenytoin; TPM topiramate; VPA valproic acid; ZNS zonisamide; day 1/2: seizure on the first/second registration day. 
1. Cortisol and global functional connectivity (averaged PLI of all channels)

\begin{tabular}{|l|cc|}
\hline \multicolumn{1}{|c|}{ Stress } & Estimate of fixed effect & $\mathbf{p}$ \\
\hline Total $(\mathbf{n}=\mathbf{1 7})$ & -0.0007 & $\mathbf{0 . 0 3}$ \\
\hline Sensitive $(\mathbf{n}=\mathbf{6})$ & -0.0020 & $<0.01$ \\
\hline Not sensitive $(\mathbf{n}=\mathbf{1 1})$ & -0.0003 & 0.46
\end{tabular}

2. Cortisol and average Strength of subject-specific channels with and without IEDs

\begin{tabular}{|c|c|c|c|c|}
\hline \multirow[b]{2}{*}{ Stress } & \multicolumn{2}{|c|}{ Strength: IED channels } & \multicolumn{2}{|c|}{ Strength: No IED channels } \\
\hline & Estimate & p & Estimate & p \\
\hline Total $(n=17)$ & -0.0007 & 0.05 & -0.0007 & 0.02 \\
\hline Sensitive $(n=6)$ & -0.0024 & $<0.01$ & -0.0019 & $<0.01$ \\
\hline Not sensitive $(n=11)$ & -0.0002 & 0.69 & -0.0003 & 0.41 \\
\hline Stress & Estimate o & effect & & \\
\hline Total $(n=17)$ & & & & \\
\hline Sensitive $(n=6)$ & & & & \\
\hline Not sensitive $(n=11)$ & & & & \\
\hline
\end{tabular}

4. Average Strength of subject-specific channels with and without IEDs and IED frequency

\begin{tabular}{|l|cc|cc|}
\hline & \multicolumn{2}{|c|}{ Strength: IED channels } & \multicolumn{2}{c|}{ Strength: No IED channels } \\
\hline \multicolumn{1}{|c|}{ Stress } & Estimate & $\mathbf{p}$ & Estimate & $\mathbf{p}$ \\
\hline Total $(\mathbf{n}=\mathbf{1 7})$ & -19.37 & $<\mathbf{0 . 0 1}$ & -11.82 & $<\mathbf{0 . 0 1}$ \\
\hline Sensitive $(\mathbf{n}=\mathbf{6})$ & -29.40 & $<\mathbf{0 . 0 1}$ & -19.81 & 0.06 \\
\hline Not sensitive $(\mathbf{n}=\mathbf{1 1})$ & -12.17 & $<\mathbf{0 . 0 1}$ & -7.27 & $\mathbf{0 . 0 3}$ \\
\hline
\end{tabular}

Table 2. Results for four different linear mixed models. All four models were also stratified for stress-sensitivity of seizures. Relations are shown for (1.) cortisol as predictor and average PLI (with lag+2) as dependent variable. Average PLI is a measure for global functional connectivity; for (2.) cortisol as predictor and average Strength $($ lag+2) of subject-specific channels with and without IEDs as dependent variable; for (3.) average PLI as predictor and IED frequency as dependent variable; and for (4.) average Strength (lag+2) of subject-specific channels with and without IEDs as predictor and IED frequency in general as dependent variable. 'IED channels' represent the column in which the average Strength of only the channels with IEDs per subject are used. These averaged Strengths per subject are then again taken together for all subjects. The same is done for 'No IED channels', but with all channels without IEDs per subject (see supplementary Figure 3 for a schematic with explanation). Bold numbers indicate a significant association. 
Supporting information (for online publication only):

Average Strength of subject-specific channels with and without IEDs.

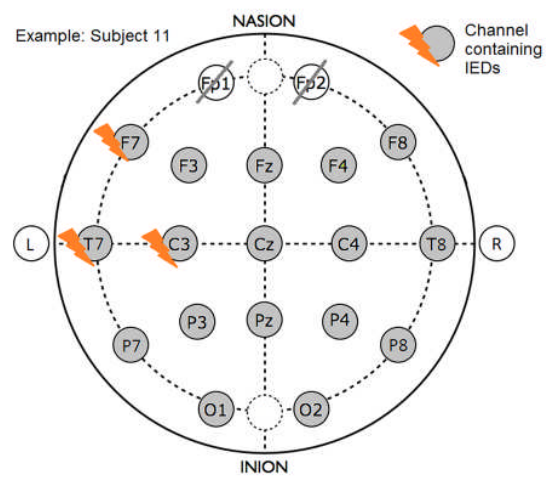

Supplementary Figure 3. Average Strength of subject-specific channels with and without IEDs. The image shows an example of subject 11, who has IEDs on channels F7, T7 and C3, as marked with the orange bolt. Strength of these three channels was then averaged to gain one value, specifically for channels with IEDs, and used for further analysis. This was done for every subject individually, for his or her specific channels containing IEDs. Strength of the remaining channels was averaged to gain a single value specifically for channels without IEDs. Fp1/2 were excluded from all analyses because of sensitivity to eye-movement artifacts. L=left. R=right.

Visualization showing the relationship between Strength and IED frequency

\section{Strength and IED frequency}

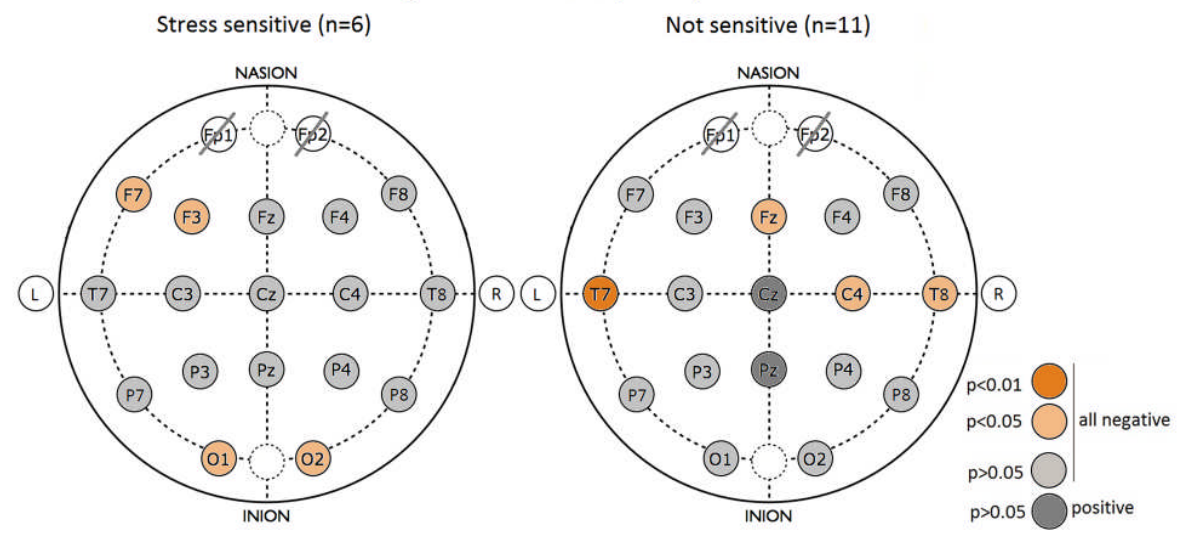

Supplementary Figure 4. Visualization of all used EEG channels, showing the relationship between Strength and IED frequency, split for those reporting stress-sensitive seizures (left) and those who do not (right), with data of all subjects per group combined. The colors indicate significance (dark orange $=p<0.01$; light orange $=p<0.05$ ), light gray has a negative relationship with $p>0.05$, dark gray has a positive relationship with $p>0.05$. L=left.

Commented [W2]: This image appears green in my Word (not readable). 
$\mathrm{R}=$ right. Only a few channels showed a significant relationship between Strength and IED frequency, both in those reporting stress-sensitive seizures and those who do not, though in different channels.

Epoch examples

Randomly selected 9 subjects, 2 epochs each.
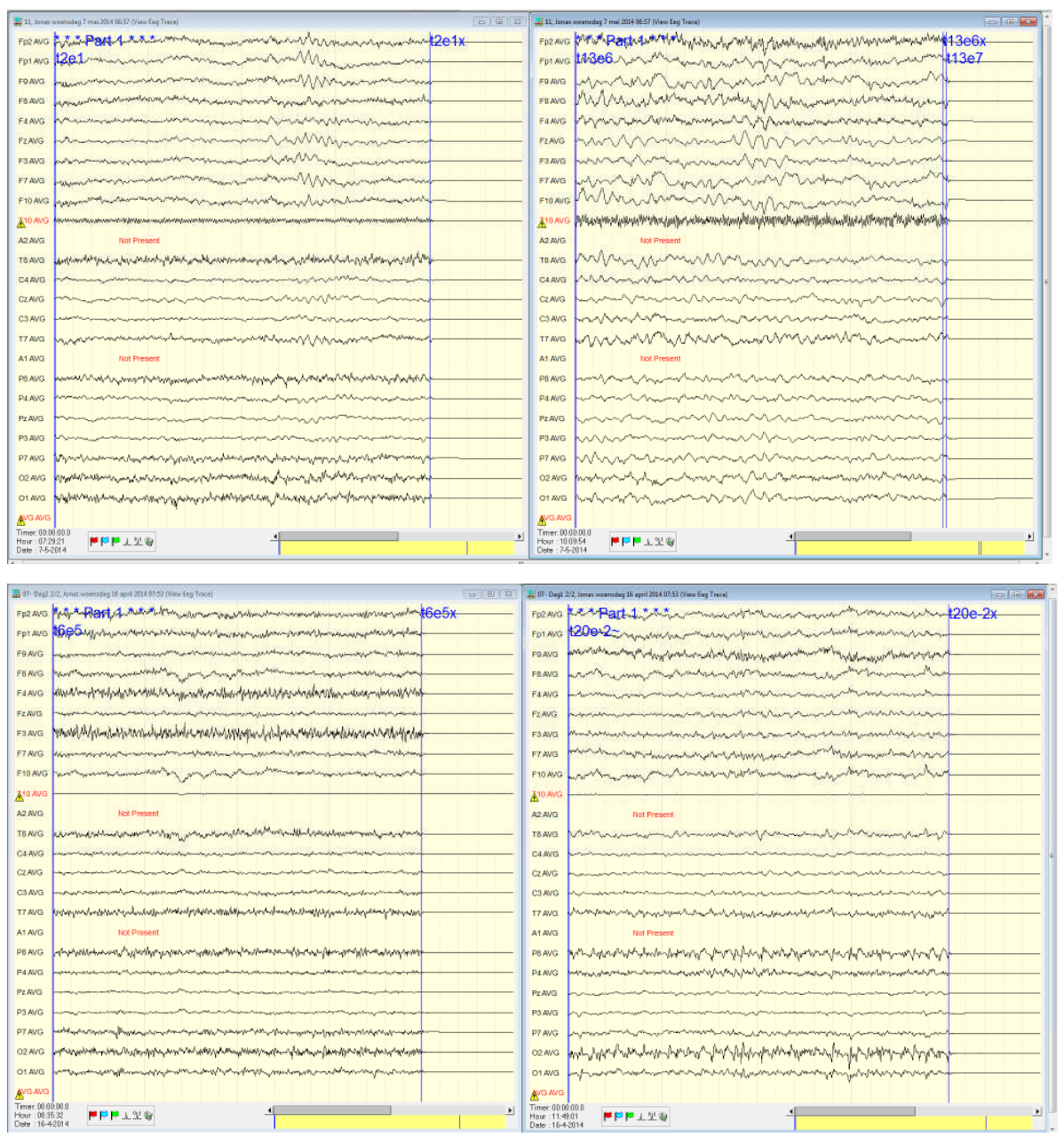

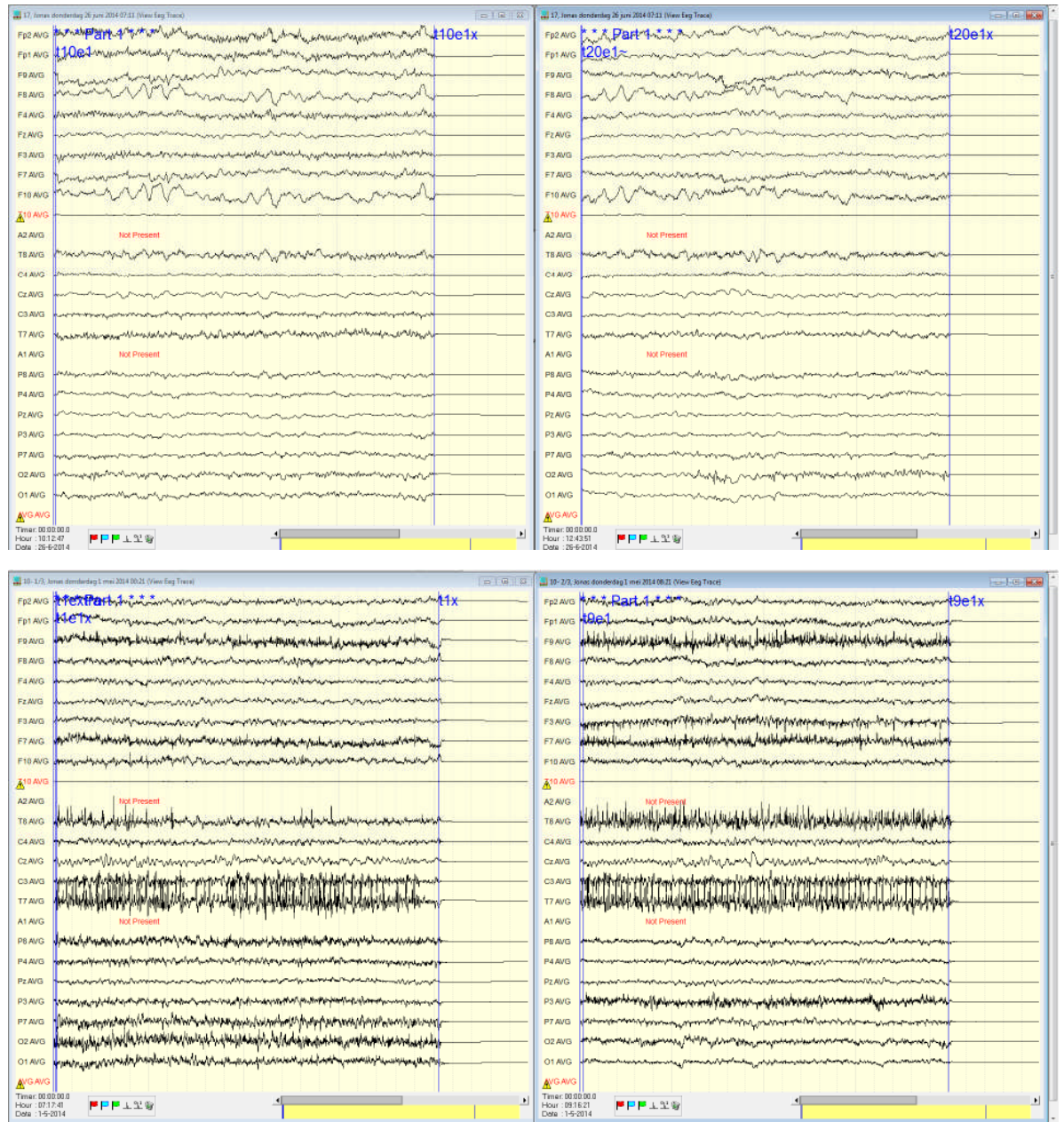

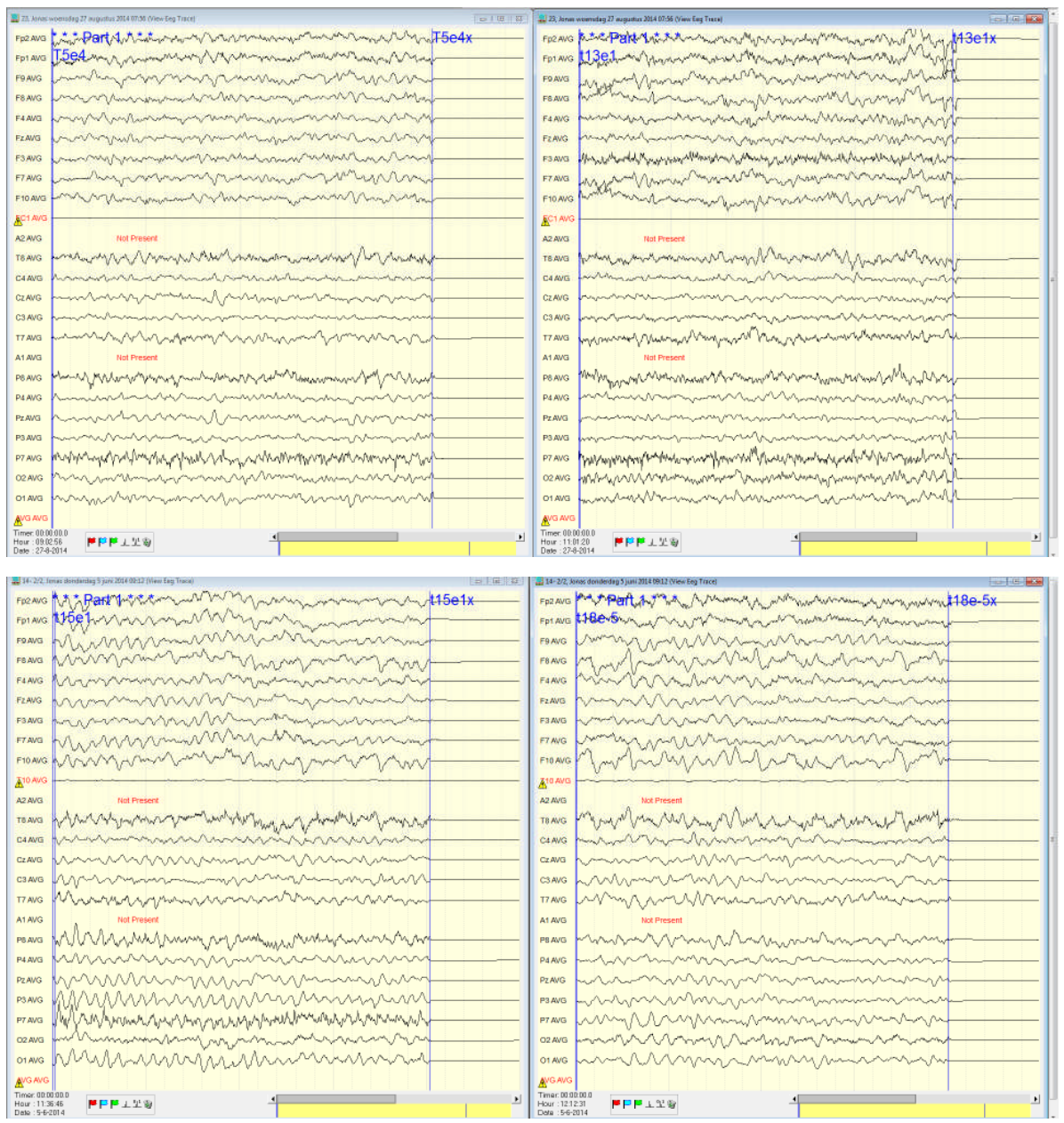

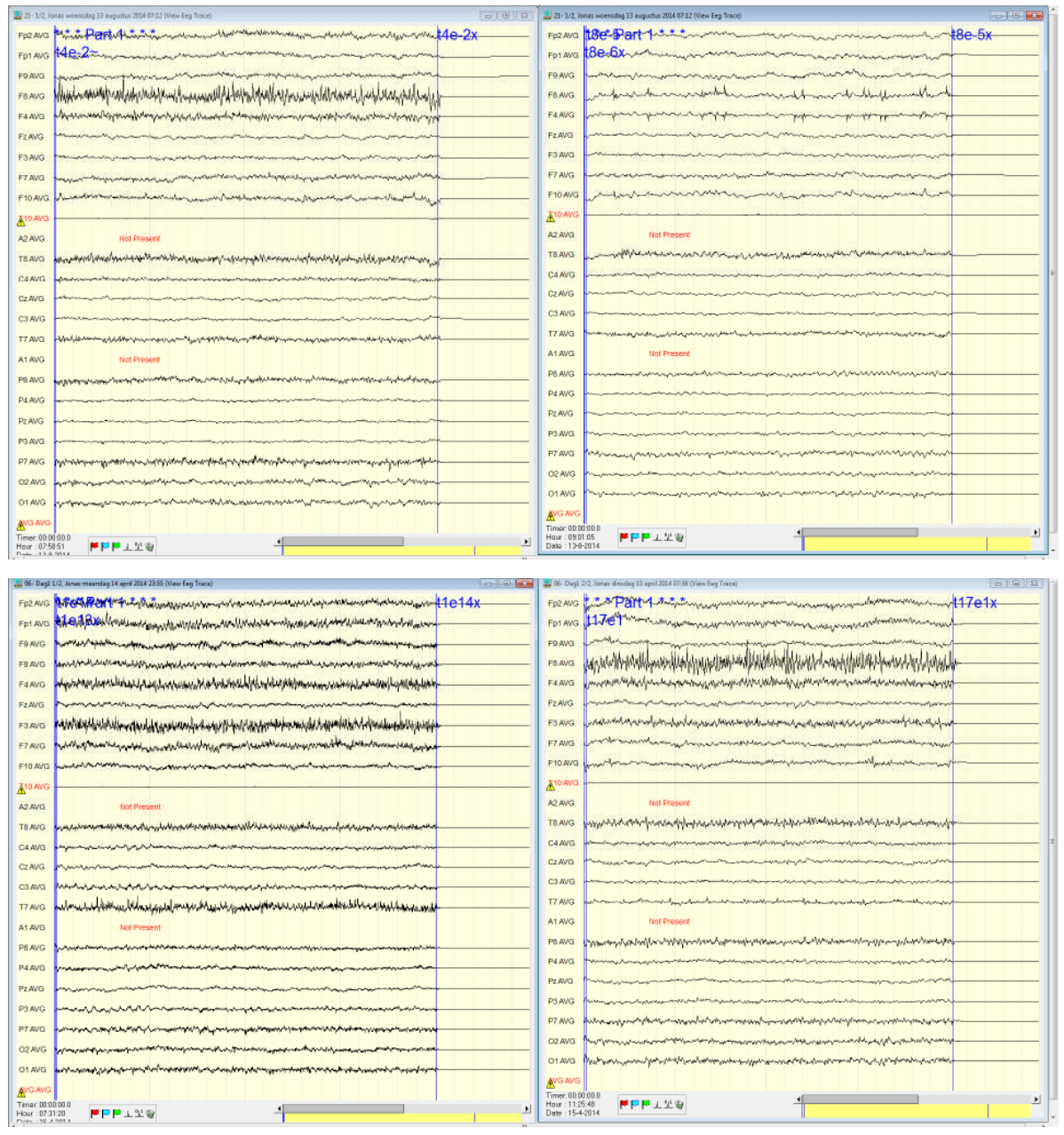


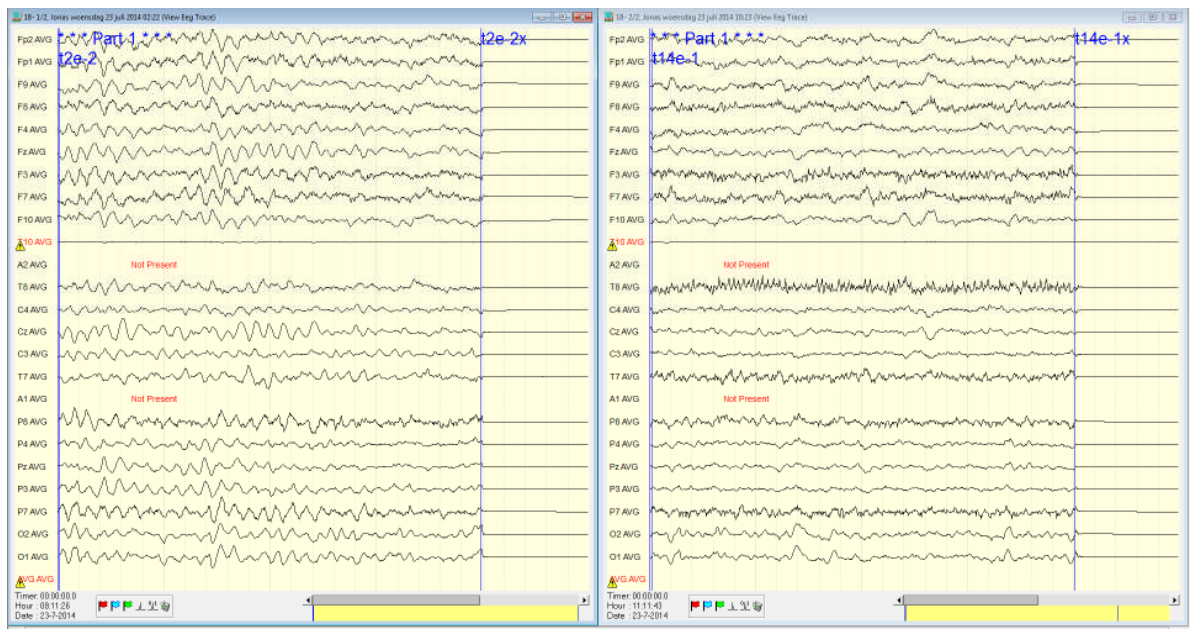

